\title{
Cat scratch disease presenting as axillary lymphadenopathy and a palpable benign mammary nodule mimicking a carcinoma
}

\author{
Larissa Cabral Marques ${ }^{[1]}$, Kátia Pincerato ${ }^{[2]}$, Adriana Akemi Yoshimura ${ }^{[1]}$, \\ Felipe Eduardo Martins Andrade ${ }^{[1]}$ and Alfredo Carlos Simões Dornellas de Barros ${ }^{[1]}$
}

[1]. Núcleo de Mastologia, Hospital Sírio Libanês, São Paulo, SP, Brasil.

[2] Laboratório de Anatomia Patológica, Hospital Sírio Libanês, São Paulo, SP, Brasil.

\begin{abstract}
We present a case involving a 74-year-old woman with cat scratch disease characterized by an enlarged and hard axillary lymph node as well as a palpable breast nodule mimicking a carcinoma. The lymph node and the breast nodule were excised. The pathologic examinations revealed granulomatous lymphadenitis with gram-negative bacilli and an intraductal papilloma. Antibiotic therapy (azithromycin) was prescribed and the patient's clinical evolution was excellent.
\end{abstract}

Keywords: Cat scratch disease. Breast. Axillary lymph node.

\section{INTRODUCTION}

Cat scratch disease (CSD) is caused by three species of Bartonella, a Gram-negative rod: Bartonella henselae (most frequent cause), Bartonella quintana, and Bartonella bacilliformis. These microorganisms have been isolated from fleas residing on infected cats, and they are usually inoculated via the scratch or bite of a kitten ${ }^{1}$.

The bacterial infection typically leads to a localized subacute lymphadenopathy in the lymphatic pathways proximal to the region of inoculation. Very rarely, injury resulting in a mammary infection is observed, followed by axillary lymphadenopathy. We report a case of a woman with axillary lymph node enlargement due to CSD, concomitant with a benign palpable breast nodule mimicking a carcinoma.

\section{CASE REPORT}

A 79-year-old woman of European descent reported a history of a longstanding breast lump and axillary lymphadenopathy for the past 6 months. On physical examination we detected a painless, hard breast nodule, measuring $2.5 \mathrm{~cm}$, located in the right upper outer quadrant and a palpable lymph node in the ipsilateral axilla, with a diameter of $1.5 \mathrm{~cm}$.

A mammogram showed parenchymal distortion and an enlarged lymph node in the right axilla. Ultrasonography

Corresponding author: Prof. Alfredo Carlos S.D. Barros.

e-mail: clinab@terra.com.br

Received 28 September 2016

Accepted 4 April 2018 revealed a regular nodule and an axillary lymph node $(1.0 \mathrm{~cm})$ with cortical thickness.

First, a core biopsy of the nodule and a fine-needle aspiration of the lymph node were performed, revealing a benign papilliferous lesion and lymphoid hyperplasia.

Because malignancy was suspected, the patient underwent surgery and the nodule and lymph node were excised. The histological findings confirmed the breast lesion as an intraductal papilloma. Granulomatous lymphadenitis and suppurative necrosis were found in the lymph node as well as pleomorphic Gram-negative bacilli upon Warthin-Starry staining specific for Bartonella henselae (Figure 1).

After the surgery, the patient received antibiotic therapy (azithromycin, 250mg/day for 5 days) and recovered completely. During a subsequent clinical investigation, we found that the patient lived with and was in frequent contact with several cats.

\section{DIscussion}

In immunocompetent individuals, the primary inoculation lesion of CSD is usually a transient vesicle, and the erythematous papule resolves within a few weeks ${ }^{2}$. In some cases, the breast can be affected with the formation of an irregular mass mimicking mastitis or cancer, which may be associated with satellite lymphadenopathy ${ }^{3-5}$. Although rare, CSD involving the breast and axilla needs to be morphologically distinguished from other common differential diagnoses to facilitate appropriate management. Primarily in immunocompromised individuals, CSD may affect many organs, with severe neurologic, visceral, and ocular manifestations ${ }^{6}$. 


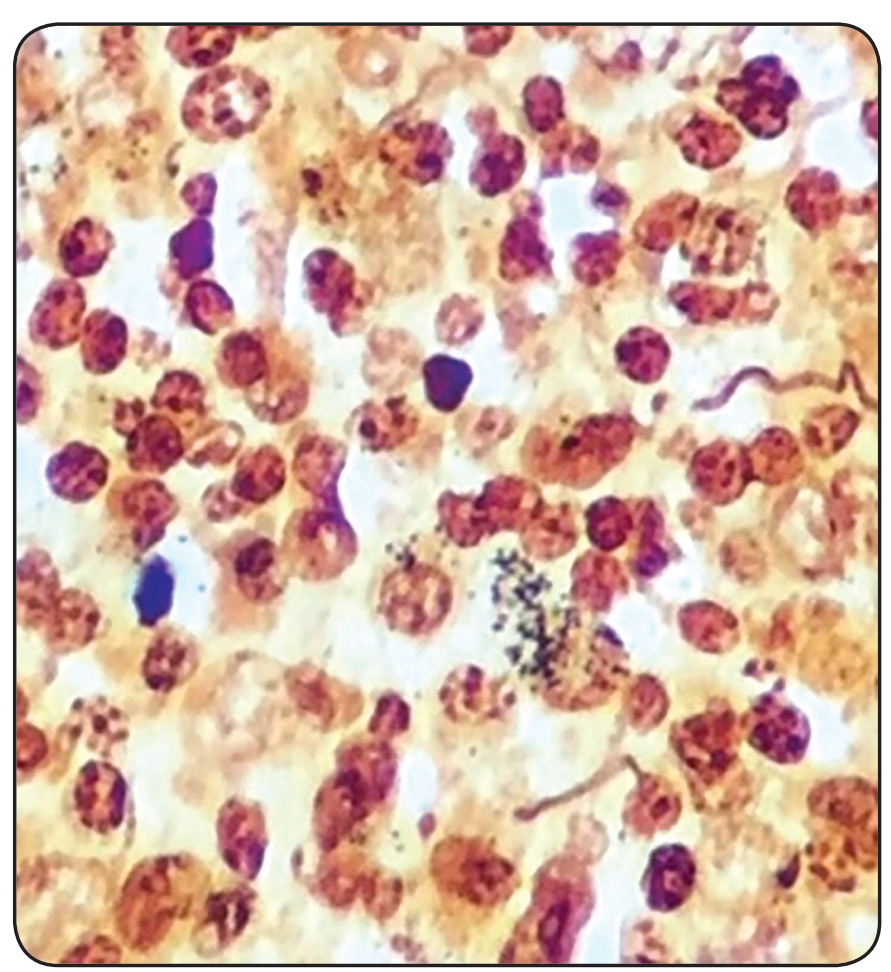

FIGURE 1: Axillary lymph node showing a group of Gram-negative bacilli stained by silver nitrate (Warthin-Starry staining) among the lymphocytes (40x).

Normally the process of the CSD is self-limited and disappears spontaneously in a few days. In certain cases, however, the breast nodule and/or the enlarged axillary lymph node persist and should be excised ${ }^{4,7}$.

Axillary lymphadenopathy may be the foremost symptom of many diseases. Ozkan et al. performed excisional biopsies and histopathological tests in 185 patients with peripheral lymphadenopathy ${ }^{7}$. Sixty-two (33.5\%) of excisional biopsy materials were benign lesions and $123(66.5 \%)$ were malignant. The benign lesions consisted of reactive hyperplasia, toxoplasmosis, necrotizing/non-necrotizing granulomatous, and CSD (four cases).

To the best of our knowledge, only approximately 10 cases of axillary lymphadenopathy following mammarian inoculation of CSD have been reported. In this case, the breast nodule corresponded to a palpable intraductal papilloma that was unrelated to the Bartonella infection. Our elderly patient did not recollect being scratched or bitten by her cats, although she used to hold them often.

CSD should be suspected when patients report frequent contact with cats along with a primary inoculation lesion and/ or regional lymphadenopathy. CSD is confirmed by the presence of silver-deposited bacilli upon Warthin-Starry staining of formalin-fixed tissues or by polymerase chain reaction of microbial deoxyribonucleic acid (DNA). Serological and culture tests can also be used for diagnostic purposes.

\section{Conflict of interest}

The authors declare that there is no conflict of interest.

\section{REFERENCES}

1. Bergmans AM, Groothedde JW, Schellekens JF, van Embden JD, Ossewaarde JM, Schouls LM. Etiology of cat scratch disease: comparison of polymerase chain reaction detection of Bartonella and Afipia felis with DNA serology and skin tests. J Infect Dis. 1995;171(4):916-23.

2. Iannace C, Lo Conte D, Di Libero L, Varricchio A, Testa A, Vigorito $\mathrm{R}$, et al. Cat scratch disease presenting as breast cancer: a report of an unusual case. Case Rep Oncol Med. 2013;2013:507504.

3. Gamblin TC, Nobles-James R, Bradley RA, Katner HP, Dale PS. Cat scratch disease presenting as breast mastitis. Can J Surg. 2005;48(3):254-5.

4. Markaki S, Sotiropoulou M, Papaspitou P, Lazaris D. Cat-scratch disease presenting as a solitary tumor in the breast: report of three cases. Eur J Obst Gynecol Rep Biol. 2003;106(2):175-8.

5. Povoski SP, Spigos DG, Marsh WL. An unusual case of cat-scratch disease from Bartonella quintana mimicking inflammatory breast cancer in a 50-year-old woman. Breast J. 2003;9(6):497-500.

6. Prutsky G, Domecq JP, Mori L, Bebko S, Matzumura M, Sabouni A, et al. Treatment outcomes of human bartonellosis: a systematic review and meta-analysis. Int J Infect Dis. 2013;17(10):e811-9.

7. Özkan EA, Göret CC, Özdemir ZT, Yanık S, Göret NE, Doğan M, et al. Evaluation of peripheral lymphadenopathy with excisional biopsy: six year experience. Int J Clin Exp Pathol. 2015;8(11):15234-9. 